\title{
On the uncertainty of digital PIV and PTV near walls
}

\author{
Christian J. Kähler · Sven Scharnowski • \\ Christian Cierpka
}

Received: 17 November 2011/Revised: 2 April 2012/Accepted: 17 April 2012/Published online: 6 May 2012

(C) The Author(s) 2012. This article is published with open access at Springerlink.com

\begin{abstract}
The reliable measurement of mean flow properties near walls and interfaces between different fluids or fluid and gas phases is a very important task, as well as a challenging problem, in many fields of science and technology. Due to the decreasing concentration of tracer particles and the strong flow gradients, these velocity measurements are usually biased. To investigate the reason and the effect of the bias errors systematically, a detailed theoretical analysis was performed using window-correlation, singe-pixel ensemble-correlation and particle tracking evaluation methods. The different findings were validated experimentally for microscopic, long-range microscopic and large field imaging conditions. It is shown that for constant flow gradients and homogeneous particle image density, the bias errors are usually averaged out. This legitimates the use of these techniques far away from walls or interfaces. However, for inhomogeneous seeding and/or nonconstant flow gradients, only PTV image analysis techniques give reliable results. This implies that for wall distances below half an interrogation window dimension, the singe-pixel ensemble-correlation or PTV evaluation should always be applied. For distances smaller than the particle image diameter, only PTV yields reliable results.
\end{abstract}

\section{Introduction}

Digital particle image velocimetry (DPIV) has become one of the most widespread techniques for the investigation of flows

C. J. Kähler $(\bowtie) \cdot$ S. Scharnowski · C. Cierpka Institute of Fluid Mechanics and Aerodynamics, Universität der Bundeswehr München,

85577 Neubiberg, Germany

e-mail: Christian.Kaehler@unibw.de because it allows for the instantaneous measurement of the flow field in a plane or volume without disturbing the flow or fluid properties (Adrian and Westerweel 2010; Raffel et al. 2007). Moreover, this technique presents the advantage that spatial flow features can be resolved and gradient-based quantities such as the vorticity can be calculated. In addition, correlation and spectral methods can be applied to analyze the velocity fields. In most cases, though, the technique is applied to efficiently measure average quantities such as mean velocity or Reynolds stress distributions because these are still the most relevant variables for the validation of numerical flow simulations and the verification or disproof of theories or models in fluid mechanics.

For a myriad of applications, the flow close to surfaces is of high interest. In biological flows for instance, the determination of the flow field around cells is very important as the behavior of cells can often be directly related to different magnitudes of the wall-shear stress. In medical flows, the wall-shear stress also plays a major role for the mechanical response of endothelial cells responsible for cardiovascular diseases (Buchmann et al. 2011; Rossi et al. 2009) or for the deposition of aerosols in lungs (Theunissen et al. 2006). In the field of process engineering, the manipulation of particles or droplets (Burdick et al. 2001) and the fluid droplet/bubble interaction (Champagne et al. 2010) requires a deep understanding of the near-wall flow phenomena. For turbulent boundary layer research, it is as well very important to resolve the near-wall flow field precisely, as the velocity profiles are typically normalized with the local mean velocity component $\bar{u}(x, y)$ or the friction velocity $u_{\tau}$ defined as:

$u_{\tau}=\sqrt{\frac{\tau_{w}}{\rho}}$

where $\rho$ is the fluid density. Since the wall-shear stress $\tau_{w}$ is given by: 
$\tau_{w}=\lim _{y \rightarrow 0} \mu \frac{\partial \bar{u}}{\partial y}$,

where $\mu$ is the viscosity of the fluid, the need to measure the mean flow gradient $\partial \bar{u} / \partial y$ precisely down to the wall is evident for a proper comparison of experimental, theoretical and numerical results (Alfredsson et al. 2011; Bitter et al. 2011; Fernholz and Finley 1996; Marusic et al. 2010; Nagib et al. 2007; Nickels 2004).

In order to resolve the flow field near walls and interfaces, it is important

1. to sample the flow motion down to the wall with appropriate tracer particles that follow the fluid motion with sufficient accuracy, as discussed in Wernet and Wernet (1994), Melling (1997) and Kähler et al. (2002),

2. to use fluorescent particles as is typically done in microfluids (Santiago et al. 1998) or a tangential illumination along a properly polished wall (Kähler et al. 2006), such that the wall reflection can be suppressed,

3. to image the particles properly with a lens or a microscope objective such that the particle signal can be well sampled on a digital camera (Adrian 1997; Hain et al. 2007; Kähler et al. 2012) and

4. to estimate the particle image displacement with digital particle imaging analysis methods (Ohmi and Li 2000; Scarano 2001; Stanislas et al. 2003, 2005, 2008).

Three different evaluation methods are well-established:

The first one is the window-correlation method that can be used to evaluate single image pairs in order to investigate instantaneous flow phenomena (Adrian and Westerweel 2010; Raffel et al. 2007) or to estimate mean flow properties as discussed in Meinhart et al. (2000). One drawback associated with this evaluation concept is the low dynamic spatial range (DSR), which is usually in the range between 20 and 250. Thus, the range of spatial scales which can be resolved with the technique is rather small. Other drawbacks result from the nonuniform particle image distribution in the vicinity of the wall and the spatial lowpass filtering that bias the location of the correlation peak in the case of near-wall flow investigations. Theunissen et al. (2008) reduced the problem of the nonuniform seeding by a vector reallocation on the basis of the gray levels within the interrogation window. Another approach was presented by Nguyen et al. (2010), who used a conformal transformation of the images at the wall and later correlated only 1D stripes of gray values on a line parallel to the surface. The velocity profile was later directly determined by a fit of the highest correlation peaks of all the lines. This method improves the resolution in the wallnormal direction at the expense of the resolution in the other direction.
The second evaluation method is the single-pixel ensemble-correlation that estimates mean flow properties from an ensemble of image pairs. Here, the spatial resolution is improved in two dimensions, and the accuracy is significantly increased. This evaluation technique was first applied by Westerweel et al. (2004) for stationary laminar flows in microfluidics. In the last years, the approach was extended for the analysis of periodic laminar flows (Billy et al. 2004), of macroscopic laminar, transitional and turbulent flows (Kähler et al. 2006) and for compressible flows at large Mach numbers (Kähler and Scholz 2006; Bitter et al. 2011). Scholz and Kähler (2006) have extended the high-resolution evaluation concept also for stereoscopic PIV recording configurations. Recently, based on the work of Kähler and Scholz (2006), the single-pixel evaluation was further expanded to estimate Reynolds stresses in turbulent flows with very high resolution (Scharnowski et al. 2011). In principle, the interrogation window size can be reduced down to a single pixel, but it was shown that the resolution is determined by the particle image size rather than the pixel size (Kähler et al. 2012). This leads to a dynamic spatial range of up to $\mathrm{DSR}=2,000$, which is an enormous improvement compared to window-correlation analysis. Although the temporal information is lost using this evaluation approach, important quantities such as the Reynolds normal and shear stresses can be extracted with improved resolution and precision, as outlined in Scharnowski et al. (2011).

The third established method for the evaluation of DPIV images is particle tracking velocimetry (PTV). Since the resolution of PTV is not affected by the digital particle image diameter, as shown in Kähler et al. (2012), this technique is often superior to correlation-based methods. However, good image quality is required for reliable measurements and, due to the random location of the velocity vectors, the application of correlation and spectral methods for the vector field analysis becomes difficult and interpolation techniques are required for the estimation of quantities based on velocity gradients. Because of the lowered seeding concentration, the dynamic spatial range is comparable to window-correlation techniques for instantaneous fields. However, if averaged data are of interest, the dynamic spatial range can be increased even beyond the range of the single-pixel evaluation to the subpixel range (Kähler et al. 2012).

The combination of PIV and PTV was also proposed by several authors to combine the robustness of correlationbased methods with the spatial resolution of tracking algorithms (Keane et al. 1995; Stitou and Riethmuller 2001). However, all evaluation methods have their respective strengths and weaknesses. Therefore, guidelines are important to know the conditions under which each method performs best. In Sects. 2 and 3, synthetic DPIV 
images are evaluated using the different methods to assess systematic errors in the vicinity of the wall. Section 4 shows the impact of the resolution limit on the estimation of the near-wall velocity for a macroscopic turbulent boundary layer flow experiment as well as for a microscopic laminar channel flow. Finally, all findings will be summarized and guidelines will be given in Sect. 5 .

\section{Synthetic test cases}

In this section, a fundamental analysis of a synthetic image set is performed for three reasons: first, it gives full control of all parameters considered for the simulation (as opposed to experiments where many uncertainties exist such as local density, temperature, viscosity, flow velocity, particle properties, illumination power and pulse-to-pulse stability, local energy density in the light sheet, imaging optics, recording medium, and bias effects due to data transfer that are unknown or cannot be precisely controlled as can be done with simulations). Second, the variation of single parameters is possible (which is often difficult to do in experiments because of the mutual dependence of the parameters like light intensity and signal-to-noise ratio, optical magnification and lens aberrations). Third, the range of the parameters can be increased beyond the experimentally accessible range (higher shear rates and turbulence levels, higher particle concentrations...).

The major drawback of the synthetic image approach is that not all physical effects can be simulated properly because of a lack of physical knowledge and the fact that each experimental setup is unique. Thus, the idealized assumptions and approximations that are used in simulations lead to deviations from experimental results. To keep the deviations small, the important physical effects must be considered, while the higher-order effects, which are below the resolution limit of the techniques, can be neglected. As this requires an a priori knowledge, experiments are always necessary to prove the main predictions and sensitivities of the simulations and to estimate the uncertainty of the simulation relative to the experiment. This will be done in Sect. 4.

In order to determine the resolution and the measurement uncertainty for velocity vectors close to the surface, synthetic DPIV images with different digital particle image diameters $D$ were generated and analyzed. The center positions of the particle images were distributed randomly to simulate a homogeneous seeding concentration. The intensity for each pixel was computed from the integral of a Gaussian function (where $D$ is four times the standard deviation) over the pixel's area, representing a sensor fill factor of one. Additionally, Gaussian noise with zero mean intensity and a standard deviation of $1 \%$ of the particle
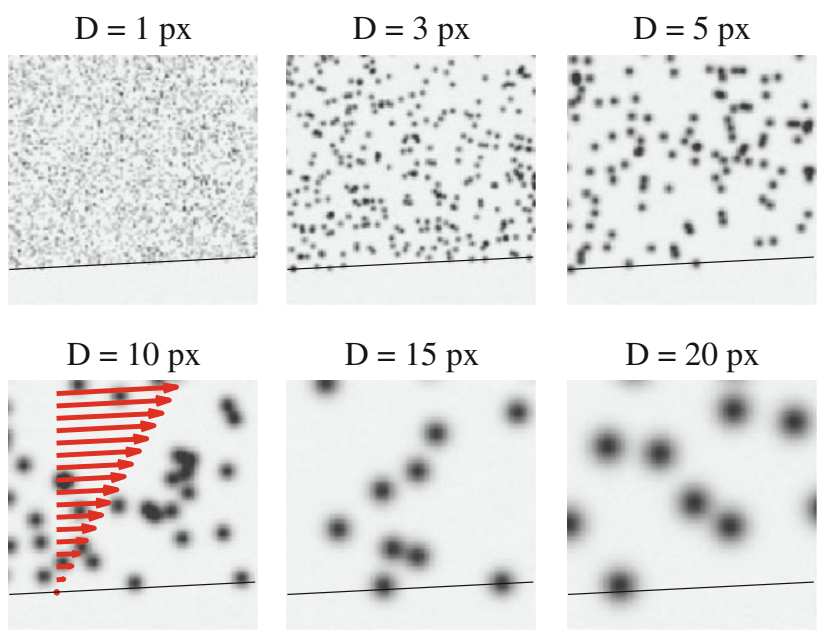

Fig. 1 Synthetic images of a near-wall boundary layer for different digital particle image diameters $D$. The surface position is indicated by the black line. $100 \times 100 \mathrm{px}$ are shown for each case

images' maximum intensity was added to simulate a signal-to-noise ratio of 100:1. The digital particle image diameter varied from $D=1 \mathrm{px}$ (which is typical for experiments in air with large observation distances) up to $D=20 \mathrm{px}$ (which is common in experiments with large magnification), as illustrated in Fig. 1. 10,000 image pairs were generated for each digital particle image diameter. The seeding concentration was about $25 \%$ for the correlation methods and $5 \%$ for particle tracking. This is to say that 25 or $5 \%$ of the image was composed by particle images. Consequently, the number of particle images changes with the digital particle image diameter, which also holds true in real experiments if the magnification is increased using a constant physical seeding concentration.

A displacement profile with a constant gradient of $\partial \Delta X^{*} / \partial Y^{*}=0.1 \mathrm{px} / \mathrm{px}$ was simulated to illustrate the effects and main sensitivities. Since spatial gradients are assumed to be negligible as long as the gradient multiplied with the interrogation window dimension is less or comparable to the particle image diameter, this implies that for a $8 \times 8 \mathrm{px}$ and $16 \times 16 \mathrm{px}$ window, the chosen gradient should be irrelevant for particle image diameters of 2-3 px, while for a $32 \times 32 \mathrm{px}$ window, it becomes slightly larger than recommended (Keane and Adrian 1992). The surface was located several pixels away from the border of the DPIV images and was slightly tilted (1:20) with respect to the image boarder to simulate the wall location at random subpixel positions. $(X, Y)$ corresponds to the image coordinates, while $\left(X^{*}, Y^{*}\right)$ denotes the wall-parallel and wallnormal coordinates, respectively. Only particle images with a random center position above the simulated surface were generated. Even though all particles are located above the wall, their images can extend into the region below the wall. This effect is illustrated in Fig. 1, where the synthetic 
Table 1 Frequently used variables and their meaning

\begin{tabular}{lll}
\hline Quantity & Symbol & Unit \\
\hline Particle diameter & $d_{p}$ & $\mu \mathrm{m}$ \\
Particle image diameter & $d_{\tau}$ & $\mu \mathrm{m}$ \\
Digital particle image diameter & $D$ & $\mathrm{px}$ \\
Dynamic spatial range & $\mathrm{DSR}$ & $\mathrm{m} / \mathrm{m}$ \\
Optical magnification & $M$ & $\mathrm{~m} / \mathrm{m}$ \\
Spatial resolution & $\mathrm{res}$ & $\mathrm{m}$ \\
Wall-parallel shift vector component & $\Delta X^{*}$ & $\mathrm{px}$ \\
Wall-normal shift vector component & $\Delta Y^{*}$ & $\mathrm{px}$ \\
Wall-normal image coordinate & $Y^{*}$ & $\mathrm{px}$ \\
Wall-shear stress & $\tau_{w}$ & $\mathrm{~N} / \mathrm{m}^{2}$ \\
Friction velocity & $u_{\tau}$ & $\mathrm{m} / \mathrm{s}$ \\
Normalized wall-normal coordinate & $y^{+}=y \cdot u_{T} / v$ & - \\
Boundary layer thickness & $\delta_{99}$ & $\mathrm{~mm}$ \\
interrogation window height & $W_{Y}$ & $\mathrm{px}$ \\
\hline
\end{tabular}

images in the near-wall region of the boundary layer are shown for different digital particle image diameters $D$. This implies a virtual velocity at negative $Y^{*}$-locations. In real experiments, this leads to the problem that the wall location cannot be estimated reliable from the velocity profile. However, for some experiments, the particle images are mirrored at the surface; in this case, the wall location can be determined precisely from the velocity profile (Kähler et al. 2006).

In the following discussion, the synthetic images are analyzed using window-correlation, single-pixel ensemblecorrelation and particle tracking velocimetry. Frequently used variables are listed in Table 1.

\section{Comparison of evaluation techniques}

\subsection{Window-correlation}

The window-correlation-based evaluation was performed for a digital particle image diameter of $D=3 \mathrm{px}$, which is close to the optimal value to achieve low RMS-uncertainties (Raffel et al. 2007; Willert 1996). Four different interrogation window sizes ranging from $8 \times 8 \mathrm{px}$ to $64 \times 64 \mathrm{px}$ were applied. The evaluation was performed using a commercial software (DaVis by LaVision $\mathrm{GmbH}$ ) with a sum-of-correlation approach. For each interrogation window size, 100 image pairs were analyzed.

The resulting wall-parallel and wall-normal shift vector components $\left(\Delta X^{*}, \Delta Y^{*}\right)$ are shown in Fig. 2 as a function of the wall-normal image coordinate $Y^{*}$. Since the simulated surface was slightly tilted, the investigation covers all subpixel distances. For larger window sizes, the typical systematic bias error becomes prominent for the estimated
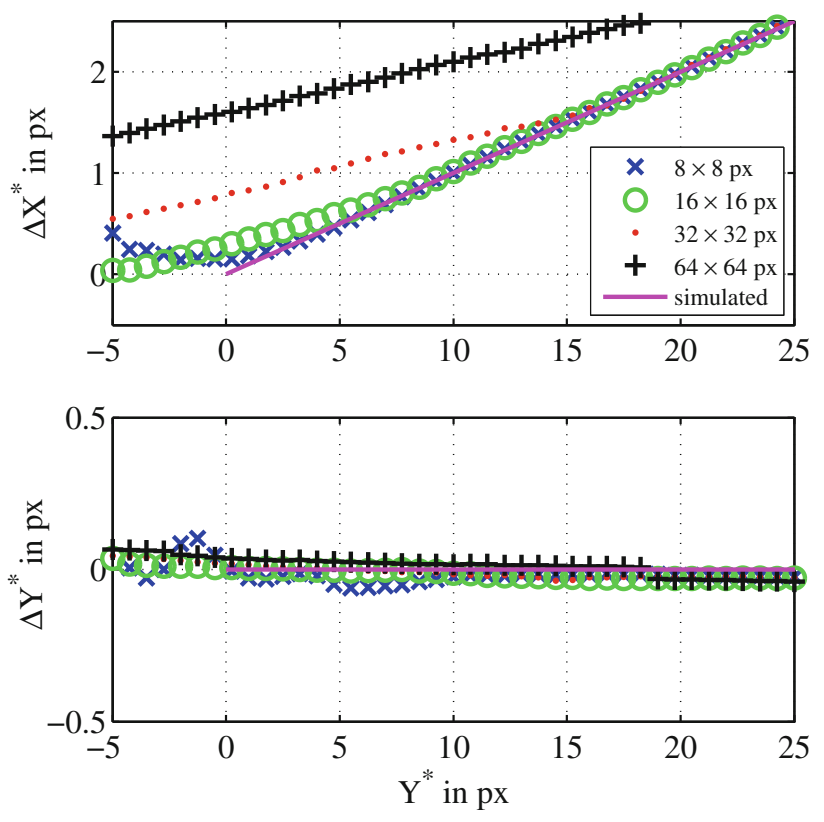

Fig. 2 Estimated displacement profiles for a simulated constant gradient in the near-wall region of a boundary layer using windowcorrelation for different interrogation windows

wall-parallel shift vector component $\Delta X^{*}$. Its magnitude is a function of the correlation window dimension in the wallnormal direction. The wall-parallel shift vector component is overestimated for locations that are closer to the wall than half the height of the interrogation window. The strong bias error is due to the fact that the mean particle image displacement, averaged over the interrogation window area, is associated with the center position of the window if no vector reallocation is performed. Therefore, even under ideal conditions (constant flow gradient, homogeneous particle image distribution, identical particle image intensity and size), no reliable near-wall displacement can be expected for distances smaller than half the interrogation window dimension in the wall-normal direction. For the $16 \times 16 \mathrm{px}$ interrogation window, for example, vectors located below $Y^{*}=8 \mathrm{px}$ are biased as expected.

The magnitude of the systematic error of the wall-parallel shift vector component $\delta \Delta X_{\text {wall }}^{*}$ at the wall position $Y^{*}=0 \mathrm{px}$ is dependent on the interrogation window height $W_{Y}$ and the mean gradient $\partial \Delta \bar{X}^{*} / \partial Y^{*}$ in the near-wall region. Without compensating for inhomogeneous particle image distribution, the following bias error occurs:

$\delta \Delta X_{\mathrm{wall}}^{*}=\frac{W_{y}}{4} \cdot \frac{\partial \Delta \bar{X}^{*}}{\partial Y^{*}}$.

In this simple model, the interrogation window centered at $Y^{*}=0 \mathrm{px}$ is, on average, only half occupied with particle images. Thus, the mean displacement within this window is equal to the wall-parallel shift vector component at $Y^{*}=W_{Y} /$ 4 for constant gradients. The vertical displacement component, shown on the bottom of Fig. 2, is not systematically 
affected since $\Delta Y^{*}$ is constant. For the case of a nonconstant shift vector gradient, an additional bias error on the estimated shift vector is expected, which scales with the profile's curvature and the interrogation window size.

It is important to note that shift vectors below the surface are computed although no particle positions were generated in this region. This is because the interrogation windows centered below the surface are still partly filled with the images of the particles located above the surface. When the wall location is known, the vectors can be easily rejected. However, in the case of a tangential illumination or fluorescent particles, the wall is not visible at all and the wall detection may become problematic. It is obvious that a proper reallocation of the vectors must be made and a suitable alignment and optimized size of the interrogation windows must be used to minimize these effects. However, this is difficult to achieve close to solid surfaces and interfaces as the ideal conditions (constant flow gradient, homogeneous particle image distribution, straight walls...) do not generally hold in real experiments.

\subsection{Single-pixel ensemble-correlation}

In the case of the single-pixel ensemble-correlation analysis, the limitations associated with the window-correlation evaluation approach do not apply as the interrogation window size can be reduced to a single pixel. However, although the flow is sampled up to a single pixel, the resolution is limited by the digital particle image diameter. It can be shown that the single-pixel analysis leads to a maximum resolution of $1.84 \mathrm{px}$, as discussed in detail in Kähler et al. (2012). The single-pixel ensemble-correlation was performed for different digital particle image diameters ranging from 1 to $20 \mathrm{px}$ using 10,000 DPIV image pairs. The correlation functions were computed using the following equation:
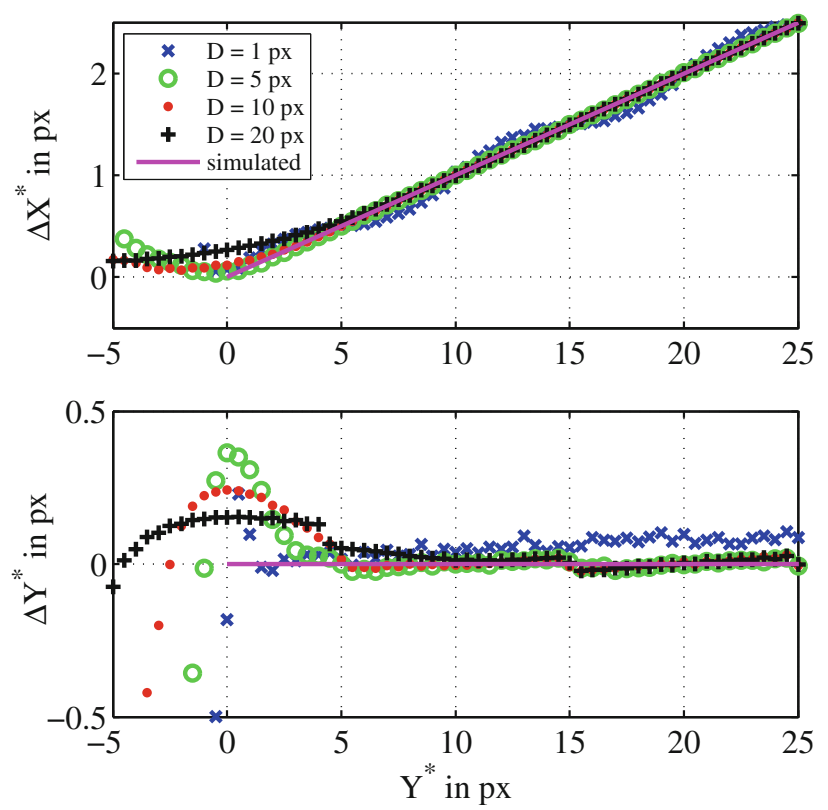

Fig. 3 Estimated displacement profiles for a simulated constant gradient in the near-wall region of a boundary layer using single-pixel ensemble-correlation for different digital particle image diameters

distribution of the first and second DPIV image of the $n$th image pair, respectively. The maximum of the correlation peak was determined using a three-point Gauss estimator for each direction.

Figure 3 shows the estimated wall-parallel and wallnormal displacement components with respect to the wall-normal distance $Y^{*}$ for $D=[1,5,10,20] \mathrm{px}$. The wall-parallel component is strongly biased for the digital particle image diameter of $D=1 \mathrm{px}$ due to the peak locking effect, which is a systematic error caused by the discretization of the measured signal (Adrian and Westerweel 2010; Fincham and Spedding 1997; Kähler 1997; Raffel et al.

$C(\xi, \psi, X, Y)=\sum_{n=1}^{N} \frac{\left[A_{n}(X, Y)-\bar{A}(X, Y)\right] \cdot\left[B_{n}(X+\xi, Y+\psi)-\bar{B}(X+\xi, Y+\psi)\right]}{\sigma A(X, Y) \cdot \sigma B(X+\xi, Y+\psi)}$

with the standard deviation given by:

$\sigma A(X, Y)=\sqrt{\frac{1}{N-1} \sum_{n=1}^{N}\left[A_{n}(X, Y)-\bar{A}(X, Y)\right]^{2}}$

where $N$ is the total number of DPIV image pairs and $n$ the corresponding control variable. $(X, Y)$ and $(\xi, \psi)$ are the discrete coordinates in physical space and on the correlation plane, respectively. $A_{n}$ and $B_{n}$ are the gray value
2007). As peak locking has a dramatic effect on the higherorder statistics of velocity profiles, according to Christensen (2004), measurements with a significant amount of small particles are not suited for high-precision flow analysis and should be considered with care. No obvious peak locking is visible for particle images larger than $D=3 \mathrm{px}$, which is in agreement with Raffel et al. (2007).

The estimated displacement profile $\Delta \bar{X}_{\text {estimated }}(Y)$ is biased due to the evaluation principle of single-pixel 


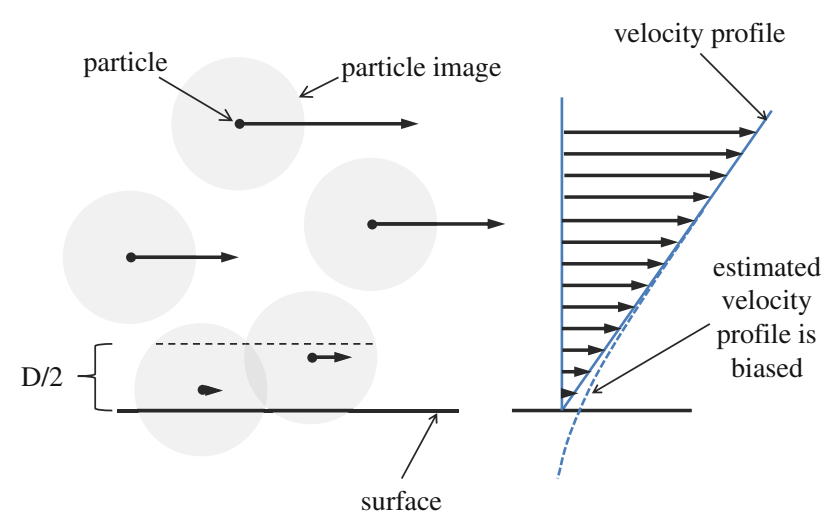

Fig. 4 Motion of particles (dark dots) and particle images (gray circles) in the near-wall region. The estimated velocity profile is biased within a distance of $D / 2$ from the surface

ensemble-correlation, as illustrated in Fig. 4. To explain this result, Eq. (4) can be transformed in an analytical expression which shows that the displacement profile is the convolution of the normalized auto-correlation of the particle image $R(D, Y)$ and the actual velocity profile $\Delta \bar{X}_{\text {true }}(Y)$ (Kähler and Scholz 2006; Scharnowski et al. 2011; Wereley and Whitacre 2007). For the wall-normal direction, this can be described in the following 1-D equation:

$\Delta \bar{X}_{\text {estimated }}(Y)=\int_{-\infty}^{\infty} R(D, Y-\psi) \cdot \Delta \bar{X}_{\text {true }}(\psi) d \psi$.

In order to estimate the difference between the true and the measured shift vector component, the integral in Eq. (6) must be solved for a specified auto-correlation and a velocity profile. The diffraction limited image of a tiny particle, in Fraunhofer approximation, can be described by a Gaussian intensity distribution function, and due to the fact that the auto-correlation function of a Gaussian function is a Gaussian function, broadened by a factor of $\sqrt{2}$, we can write:

$R(D, Y)=\frac{2}{D \sqrt{\pi}} \cdot e^{-\frac{4 \cdot Y^{2}}{D^{2}}}$

The normalization by the factor in front of the exponential term in Eq. (7) ensures that the integral over $Y$ equals one. The simulated displacement profile with constant gradient used in this analysis can be described as follows:

$\Delta \bar{X}^{*}$ true $\left(Y^{*}\right)=\Theta\left(Y^{*}\right) \cdot Y^{*} \cdot \frac{\partial \Delta \bar{X}^{*} \text { true }}{\partial Y^{*}}$,

where $\Theta\left(Y^{*}\right)$ represents the Heaviside step function. Using Eqs. (7) and (8) for the normalized auto-correlation and the simulated displacement profile, respectively, results in the following estimated shift vector profile:

$$
\Delta X_{\text {estimated }}^{*}\left(Y^{*}\right)=\frac{\partial \Delta \bar{X}^{*}}{\partial Y^{*}} \cdot\left[\frac{D}{4 \sqrt{\pi}} \cdot e^{-\frac{4 \cdot Y^{* 2}}{D^{2}}}+\frac{1}{2}\left(1+\operatorname{Erf}\left[\frac{2 \cdot Y^{*}}{D}\right]\right)\right] .
$$

For large distances from the wall $\left(Y^{*} \gg D\right)$, the Gaussian in Eq. (9) becomes zero and the error function becomes one. Thus, the estimated shift vector is not biased in the case of homogeneous seeding and constant gradients:

$\Delta X_{\text {estimated }}^{*}\left(Y^{*} \gg D\right)=\frac{\partial \Delta \bar{X}^{*}}{\partial Y^{*}} \cdot Y^{*}=\Delta X_{\text {true }}^{*}\left(Y^{*}\right)$.

On the other hand, the velocity profile is strongly biased in the vicinity of the wall. The magnitude of the systematic error of the wall-parallel shift vector component $\delta \Delta X_{\text {wall }}^{*}$ from Eq. (9) at the position of the wall at $Y^{*}=0 \mathrm{px}$ is proportional to the digital particle image diameter $D$ and the mean gradient $\partial \Delta \bar{X}^{*} / \partial Y^{*}$ in the near-wall region:

$\delta \Delta X_{\text {wall }}^{*}=\Delta X_{\text {estimated }}^{*}\left(Y^{*}=0\right)=\frac{D}{4 \sqrt{\pi}} \cdot \frac{\partial \Delta \bar{X}^{*}}{\partial Y^{*}}$.

The analytical bias error of the wall-parallel shift vector component is in good agreement with the simulated values within the shift vector profiles shown in Fig. 3. Thus, from the theoretical point of view, it seems possible to compensate for the bias error produced by the correlation procedure. However, in the case of real DPIV images, the inhomogeneous particle image distribution and the real shape of the velocity profile are not generally known. Furthermore, the deconvolution of the discrete values computed from digital images is a mathematically ill-posed problem.

It is interesting to note that the wall-normal component $\Delta Y^{*}$ is also biased in the near-wall region (Fig. 3, bottom) because the particle images further away from the wall broaden the correlation peak only on the side facing away from the surface. In the case of flows with constant gradients and ideal conditions (homogeneous particle image distribution...), this effect is averaged out as long as the particle is at least $Y^{*}>D / 2$ away from the wall. In case of instantaneous flow measurements with window-correlation PIV, this effect cannot be avoided and results in an increased random error.

For negative $Y^{*}$ values, the effect causing the systematic motion in the positive $Y^{*}$ direction can reverse, see Fig. 3 at $D=10$ and $Y^{*}<-2.5 \mathrm{px}$. This bias error is due to the normalization of the correlation by its variance. For regions lower than $Y^{*}<D / 2$, the gray value distribution over the ensemble can only have values lower than the maximum intensity and only for particles that are within a range of $Y^{*}<D$. If these ensembles are correlated with ensembles further away $(+)$ from the wall, more signal peaks (due to the uniform seeding) with large magnitudes up to the maximum intensity are present. Some of them might give a 


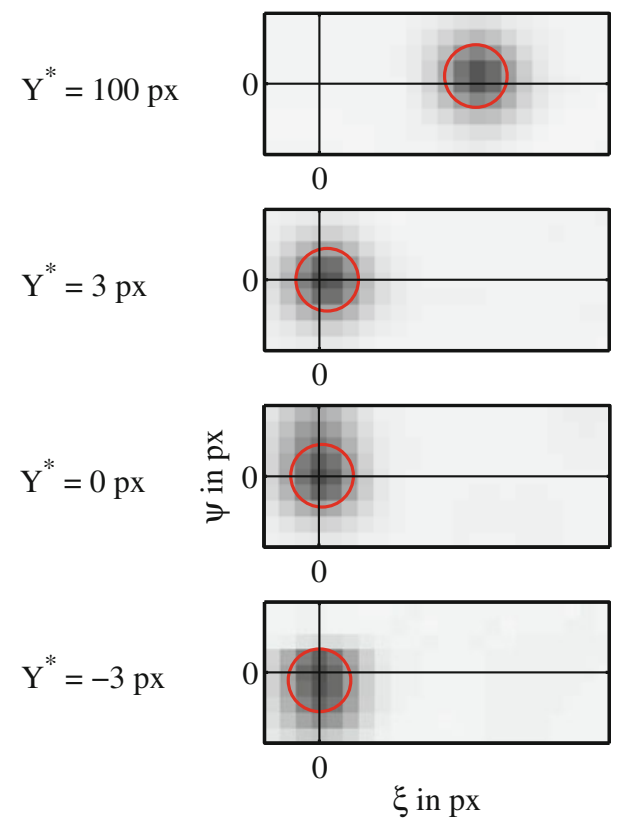

Fig. 5 Correlation planes in the vicinity of the wall for $D=5 \mathrm{px}$ and $\partial \Delta \bar{X}^{*} / \partial Y^{*}=0.1 \mathrm{px} / \mathrm{px}$

good correlation. However, the peak is averaged by its variance that is quite high for these signals, and thus, the correlation value itself is low.

If the aforementioned gray value distribution is correlated with an ensemble closer to the wall (-), there are no signals from other particle images, since there are no particles below the wall. In addition, the signal gets lower as it approaches the wall and thus the already good correlation is normalized by a small variance. The peaks closer to the wall $(-)$ are therefore more pronounced than the peaks further away $(+)$ and the wall-normal component reverses. Some correlation peaks in the vicinity of the wall are shown in Fig. 5.

Nevertheless, $\tau_{w}$ can be estimated directly from the first values above the wall that are not biased according to Eq. (9). Now the experimenter has to determine whether the positions of these first reliable vectors are close enough for the estimation of the wall-shear stress, that is they belong to the viscous sublayer of a turbulent boundary layer flow, for instance, or not and whether the mean particle image displacement is large enough for reliable measurements with low uncertainty.

\subsection{Particle tracking velocimetry}

The previous analysis shows that the resolution of windowcorrelation and single-pixel ensemble-correlation is limited by the window size and the digital particle image diameter, respectively. Thus, the question arises whether the resolution can be further enhanced using PTV algorithms that are

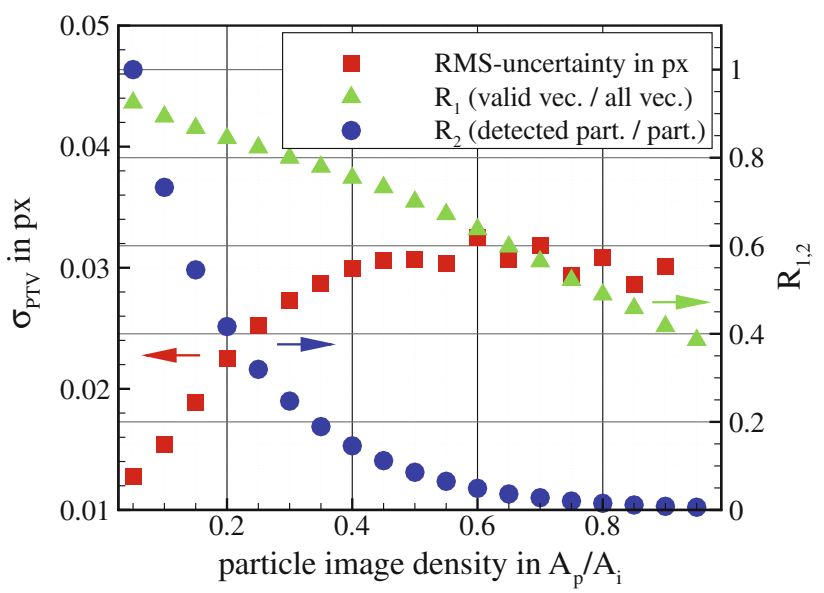

Fig. 6 RMS-uncertainty and ratio of valid vectors to total vectors and detected particles to the number of generated particles for increasing particle image density for $D=5 \mathrm{px}$

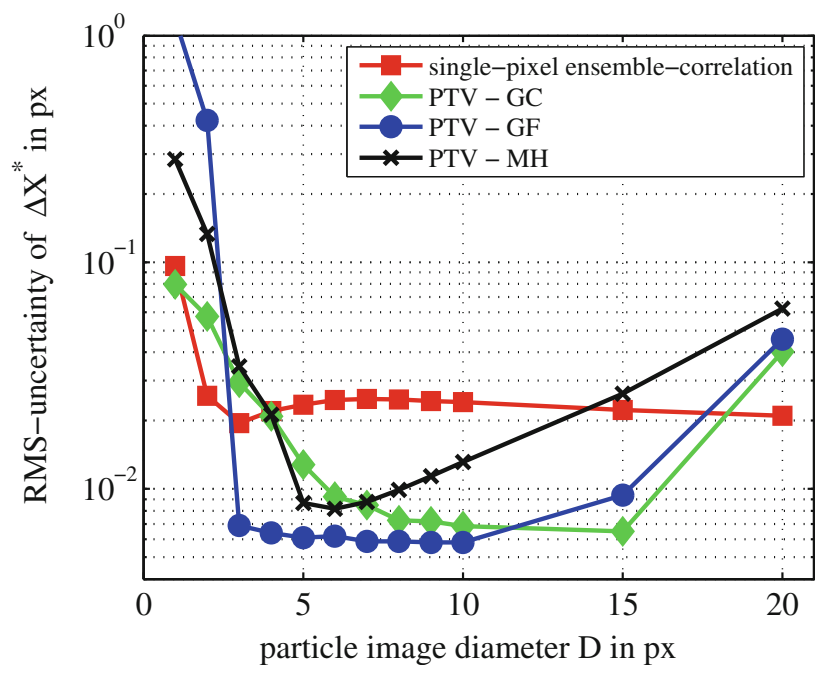

Fig. 7 Random error of the estimated displacement using PTV and single-pixel ensemble-correlation

not based on image correlations. For elaborate particle tracking algorithms, high seeding concentrations are no longer a limitation (Ohmi and $\mathrm{Li}$ 2000). However, the main source of random errors are associated with low SNR and overlapping particle images. As illustrated in Fig. 6, the RMS-uncertainty $\sigma_{\mathrm{PTV}}$ of the displacement estimation increases with increasing seeding concentration as opposed to the number of valid vectors $R_{1}$ and the ratio of detected particles to the number of generated particles $R_{2}$ which decrease strongly. Therefore, the particle image density was reduced to $5 \%$ (illuminated area) in order to avoid an increasing measurement uncertainty caused by overlapping particle images. Figure 7 shows the random error further away from the wall, computed with single-pixel ensemblecorrelation and PTV, with respect to the digital particle 

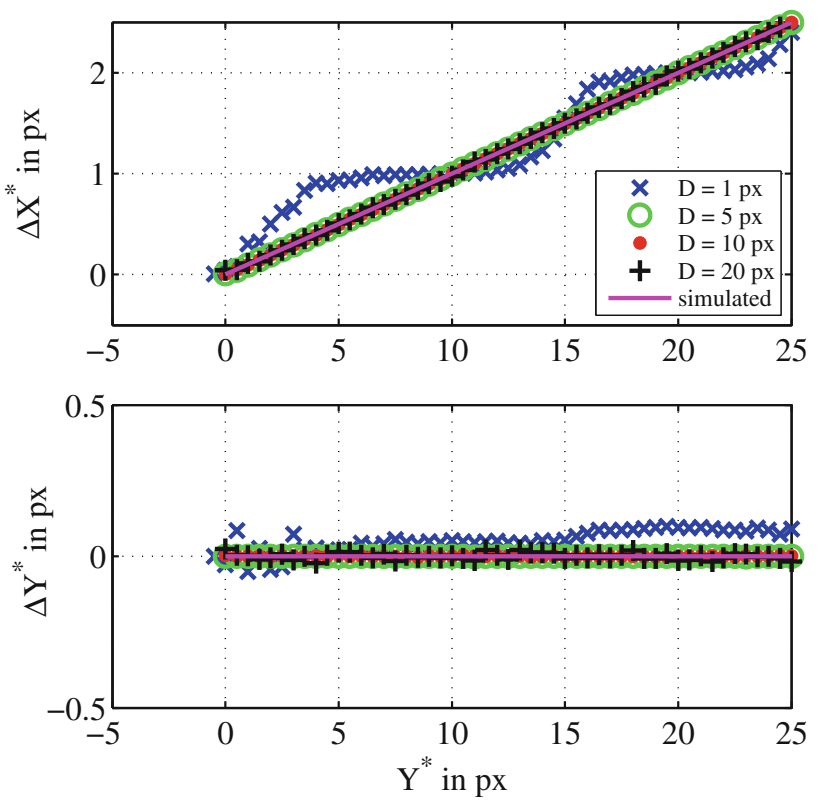

Fig. 8 Estimated displacement profiles for a simulated constant gradient in the near-wall region of a boundary layer using PTV (GCcentroid estimation) for different digital particle image diameters

image diameter $D$. The random error is fairly constant below $0.03 \mathrm{px}$ in the case of single-pixel ensemble-correlation for $D=2 \ldots .20 \mathrm{px}$. A much more pronounced influence of the digital particle image diameter can be seen for the PTV results. In addition, the big difference of the error using different centroid estimation methods is highlighted by the three curves. The line denoted as 'PTV-GC' uses a correlation of a Gaussian with the particle image, the curve denoted as 'PTV-GF' shows results of a 2D Gaussian fit for the center estimation, and PTV-MH indicates a Mexican Hat wavelet algorithm to detect the particle center. In general, the PTV-GC approach shows good results for particle images larger than $D>4 \mathrm{px}$. The error is lower than $0.01 \mathrm{px}$ for $D=6 \ldots 15 \mathrm{px}$. The Gaussian fit estimation shows good results for a much larger range of diameters, which is related to the fact that the synthetic images resemble a Gaussian intensity distribution integrated on the pixel grid of the camera sensor. In this case, the error is lower than $0.01 \mathrm{px}$ for $D=3 \ldots 15 \mathrm{px}$.

The evaluation was again performed for digital particle image diameters ranging from 1 to $20 \mathrm{px}$. Figure 8 shows the estimated wall-parallel and wall-normal displacement components with respect to the wall-normal distance $Y^{*}$ for $D=[1,5,10,20]$ px. It is clearly visible that in the case of $D=1 \mathrm{px}$, the PTV evaluation causes extreme peak locking. This is due to the fact that the position of particle images seems to be at the center of a pixel for very small digital particle image diameters. For the data presented here, the center was estimated to be at the highest correlation of the particle images with a Gaussian function

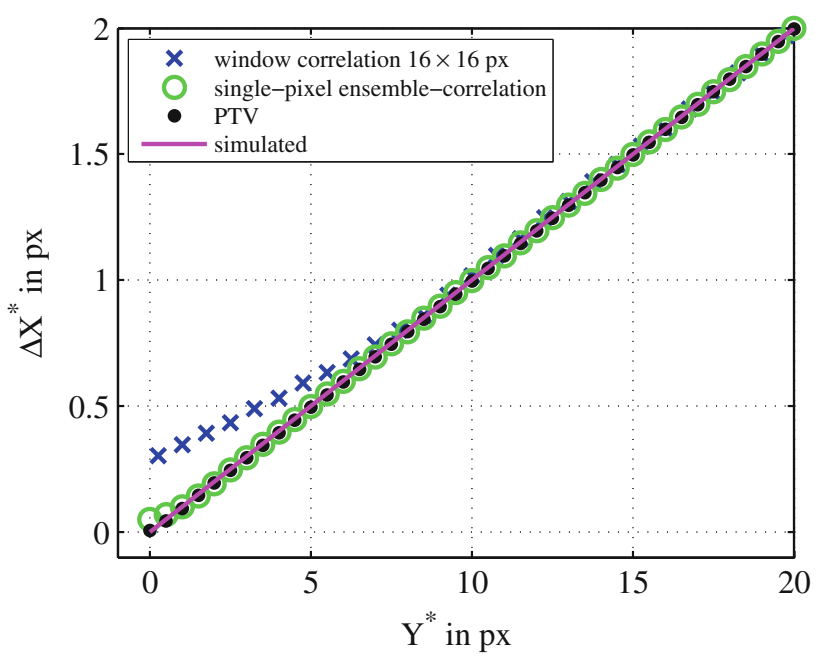

Fig. 9 Comparison of the different evaluation methods: profile of the estimated horizontal shift vector component computed using windowcorrelation, single-pixel ensemble-correlation and PTV. The particle image diameter was $D=5 \mathrm{px}$

(denoted as PTV-GC in Fig. 7). In principle, for each type of particle images, a suitable function can be found to determine the particle image center. A Mexican hat function performed particularly well for microfluidic investigations (Cierpka et al. 2010). However, for the synthetic images used here, a Gaussian fit requires much more computational time but is better suited as can be seen in Fig. 7, indicated by PTV-GF. The results are unsatisfactory only for small particle images. For particle images larger than $D=3 \mathrm{px}$, the PTV approach works well with a manifold of different particle detection algorithms (Cardwell et al. 2011). Despite the random errors, it should be noted that no bias error appears, as observed for windowcorrelation and single-pixel ensemble-correlation close to the wall. The near-wall displacement is not overestimated and no displacement vectors are computed for $Y^{*}<0 \mathrm{px}$ since no particle images are present in this region. Especially in microfluidics, where large particle images are typically present, PTV is often better suited for the velocity estimation, as discussed in Cierpka and Kähler (2012).

\subsection{Near-wall gradient}

The comparison of the three evaluation techniques showed significant differences for the estimated shift vector profiles of the synthetic near-wall flow with constant gradient. Figure 9 summarizes the results using a digital particle image diameter of $D=5 \mathrm{px}$ for window-correlation, single-pixel ensemble-correlation and particle tracking velocimetry. In the case of window-correlation, an interrogation window size of $16 \times 16 \mathrm{px}$ was used. It can clearly be seen from Fig. 9 that the biased region extends to $Y^{*} \approx 8 \mathrm{px}$ for window-correlation, whereas the biased 


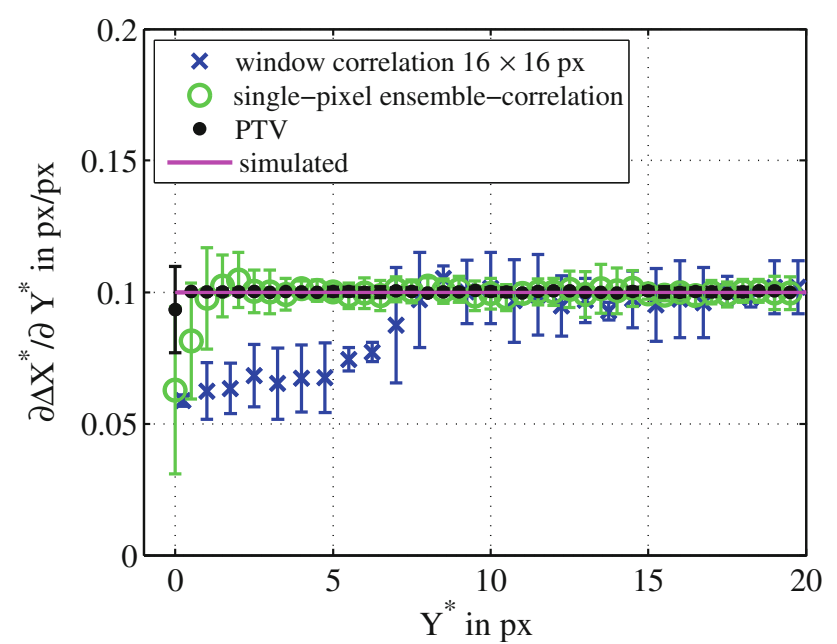

Fig. 10 Profiles of the estimated gradient from Fig. 9 computed using window-correlation, single-pixel ensemble-correlation and PTV

region and the bias error itself are much smaller in the case of single-pixel ensemble-correlation. Furthermore, the PTV results appear bias-free.

For the estimation of the wall-shear stress, the mean near-wall gradient is determined from the shift vector profiles. Figure 10 shows the gradient $\partial \Delta X^{*} / \partial Y^{*}$ of the profiles from Fig. 9 with respect to the wall-normal distance. Each point in Fig. 10 represents the slope of a linear fit-function applied to three points of the profile. The error bar corresponds to the $95 \%$ confidence interval of the fit parameters. Again, the size of the biased region strongly depends on the applied evaluation technique. It is interesting to note that the very first data point is also biased in the case of PTV. This is due to the uncertainty in the estimation of the particle image positions: Particles slightly further away from the surface, with slightly higher velocity, might be associated with the wall location, but, on the other hand, no particles can be found below the surface. Based on these results, it can be concluded that for the estimation of the near-wall gradient, the only shift vectors that can be used are those that have a distance normal to the surface larger than

- half the interrogation window size in the case of window-correlation (without vector reallocation),

- half the particle image diameter in the case of singlepixel ensemble-correlation and

- the uncertainty of the estimated particle image position in the case of PTV.

Additionally, the selected shift vectors must belong to the viscous sublayer such that the normalized wall distance $y^{+}$ is not larger than five and the displacement must be large enough for a reliable estimation as the slope of the gradient depends on both the location and velocity at the same time.
Therefore, it is often more accurate to use particle imaging techniques than LDV or hotwire probes for the analysis of flows with strong velocity gradients. LDV or hotwires are more precise than single-pixel DPIV and PTV in estimating the velocity; however, the error in estimating the exact location of the measurement volume (or the particle position inside the volume) is much larger. For singlepixel DPIV and PTV, in contrast, the locations of the particles are precisely known from the image analysis.

\section{Experimental verification}

To prove the findings of the previous section, three experiments were performed at magnifications of $M=0.1$, 2.2 and 12.6 to cover the imaging range from the macroscopic to the microscopic domain. Experiments at low-magnification realize a large field of view with small particle images and a high particle image density, in general. Thus, low-magnification experiments are well suited for single-pixel ensemble-correlation or window-correlation depending on the number of image pairs. Increasing the magnification results in larger particle images and does not gain much spatial resolution in case of correlationbased DPIV evaluation, as discussed in detail in Kähler et al. (2012). However, PTV evaluation results in increased spatial resolution in the case of a large number of image pairs, as the resolution is only limited by the error in the determination of the particle image location and the particle image displacement.

\subsection{Large field DPIV investigation at low magnification}

The first experiment was performed in the large-scaled Eiffel type wind tunnel located at the Universität der Bundeswehr München. The facility has a 22-m-long test section with a rectangular cross-section of $2 \times 2 \mathrm{~m}^{2}$. The flat plate model is composed of coated wooden plates with a super-elliptical nose with a $0.48-\mathrm{m}$-long semi-axis in the stream-wise direction. The flow was tripped $300 \mathrm{~mm}$ behind the leading edge of the plate by a sandpaper strip. Three DEHS particle seeders producing fog with a mean particle diameter of $d_{P} \approx 1 \mu \mathrm{m}$ (Kähler et al. 2002) were used to sample the flow. The light sheet for illuminating the particles was generated by a Spectra Physics Quanta-Ray PIV 400 Nd:YAG double-pulse laser. The light sheet thickness was estimated to be $500 \mu \mathrm{m}$. For the flow measurements, a PCO.4000 camera (at a working distance of $1 \mathrm{~m}$ ) in combination with Zeiss makro planar objective lenses with a focal length of $100 \mathrm{~mm}$ was used. The results presented here are taken at $6 \mathrm{~m} / \mathrm{s}$ free stream velocity, which corresponds to a Reynolds number based on 
momentum thickness at the measurement location of $R e_{\delta_{2}}$ $=4,600$. A detailed description of the experimental setup is outlined in Dumitra et al. (2011). In order to resolve the complete boundary layer velocity profile, a large field of view was selected that extends almost $250 \mathrm{~mm}$ in the wallnormal direction. The particle image concentration is close to $100 \%$ (illuminated area), and the digital particle image diameter is in the range of $D \approx 2 \ldots 3 \mathrm{px}$. These conditions are well suited for the single-pixel ensemble-correlation, according to Fig. 7 and the analysis in Sect. 3.2. On the other hand, the small particle image size and the high concentration, which causes a large amount of overlapping images, would lead to large errors for PTV, according to Fig. 6. Thus, this low-magnification data set is evaluated using single-pixel ensemble-correlation.

Figure 11 shows the boundary layer velocity profile evaluated with single-pixel ensemble-correlation. The profile represents the stream-wise velocity, averaged in the stream-wise direction over a length of $x=7.6 \mathrm{~mm}$. In total, 2,300 double frame images were processed. For the current magnification, the resolution in the wall-normal direction is $230 \mu \mathrm{m}$ which gives a spatial dynamic range of 1,000 independent velocity vectors. In normalized wall units, the resolution corresponds to $y^{+}=y \cdot u_{T} / v \approx 4$ and the first data points are already at the limit of the viscous sublayer $\left(y^{+} \leq 5\right)$. Due to the large field of view and the high dynamic spatial range (DSR), which corresponds to the number of independent vectors in each direction, the boundary layer thickness could be reliably determined to be $\delta_{99}=130 \pm 4 \mathrm{~mm}$. Thus, the relative uncertainty in estimating this quantity is about $3 \%$. However, precisely measuring the mean flow gradient $\partial \bar{u} / \partial y$ down to the wall

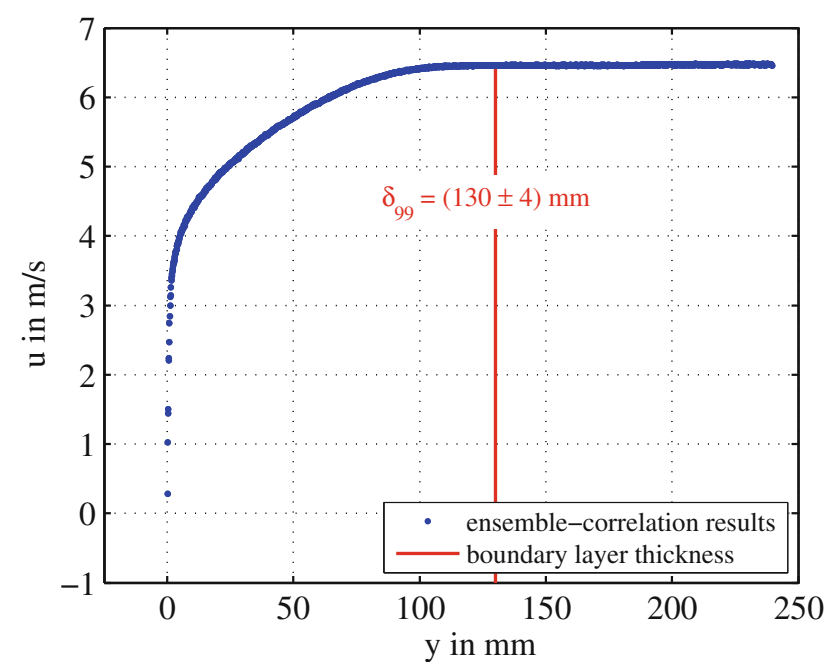

Fig. 11 Estimated velocity profile of a turbulent boundary layer using single-pixel ensemble-correlation with a resolution of res = $230 \mu \mathrm{m}$ and a dynamic spatial range of DSR $>1,000$ in the direction normal to the wall is not possible since the viscous sublayer $\left(y^{+}<5\right)$ is not resolved sufficiently, as shown in the normalized semilogarithmic representation in Fig. 12. Using the Clauser method (Clauser 1956), the wall-shear stress can be estimated from the logarithmic region of the boundary layer profile by means of the following equation:

$\frac{\bar{u}(y)}{u_{\tau}}=\frac{1}{\kappa} \cdot \ln \left(\frac{y \cdot u_{\tau}}{v}\right)+B$

The uncertainty of this approach is that the value of the constants $\kappa$ and $B$ are generally unknown. $\kappa$ and $B$ depend on the Reynolds number, the pressure gradient of the flow and other parameters. For flows along smooth walls and zero pressure gradient, typical values are $\kappa=0.41$ and $B=5.1$. In this case, $u_{\tau}$ becomes $0.248 \mathrm{~m} / \mathrm{s}$ and the wallshear stress $0.076 \mathrm{~N} / \mathrm{m}^{2}$. Here, the estimation of $\kappa$ and $B$ from the logarithmic region of the velocity profile results in different values when using a fit-function, see Fig. 12, as $\partial p / \partial x$ is not exactly zero. To avoid the uncertainty of this Clauser approach in general, a higher magnification is necessary to directly resolve the wall-shear stress according to Eqs. (1) and (2).

\subsection{Long-range microscopic DPIV}

In order to resolve the wall-normal gradient within the viscous sublayer, the magnification was increased using a long-distance microscope system (K2 by Infinity). The light sheet thickness and the working distance were again $500 \mu \mathrm{m}$ and $1 \mathrm{~m}$, respectively. The magnification was set to $\mathrm{M} \approx 2.2$ resulting in particle images with a size of $D \approx 8 \ldots 10$ px. Due to the high magnification, the seeding

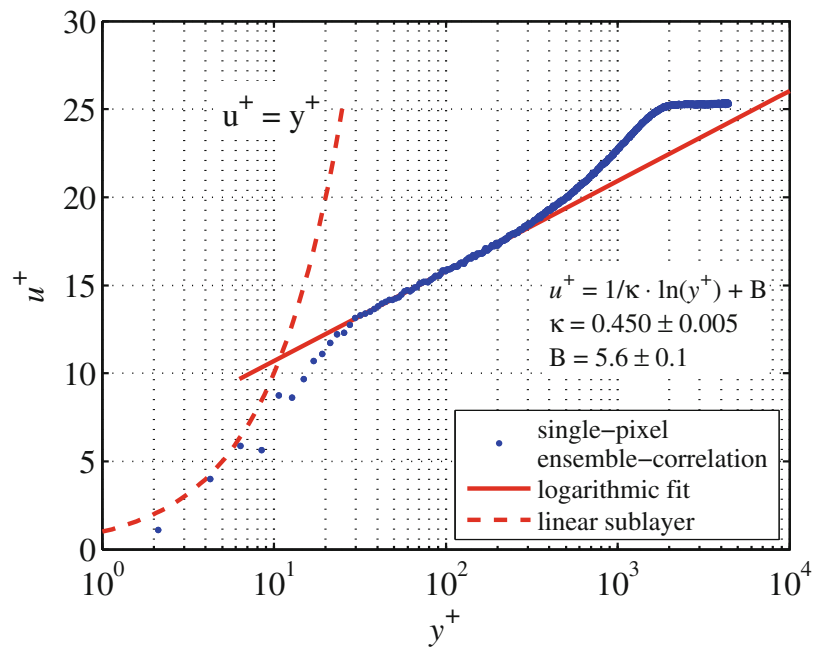

Fig. 12 Velocity profile of the turbulent boundary layer from Fig. 11 normalized with $u_{\tau}=0.256 \mathrm{~m} / \mathrm{s}$. The viscous sublayer is not sufficiently resolved. The logarithmic region was approximated by a fit-function 
concentration is rather sparse. The density of particle images, in terms of illuminated area, was less than $3 \%$. At this seeding density, a huge number of image pairs would be required for single-pixel ensemble-correlation. Additionally, the large particle images limit the resolution to $>6$ pixel, according to Kähler et al. (2012), which corresponds to $25 \mu \mathrm{m}$. On the other hand, PTV evaluation seems to be well suited for this kind of data, since the resolution is not limited by the particle images size and the low density allows for reliable detection and tracking of the particle images.

A total number of 13,000 double frame images were processed with a PTV algorithm, resulting in about 335,000 valid vectors. The velocity vectors were then averaged in the stream-wise direction (over $2.1 \mathrm{~mm}$ ) with a slot width of $20 \mu \mathrm{m}$ in the wall-normal direction. This results in a dynamic spatial range of DSR $=750$ independent velocity vectors. The resulting profile is shown in Figs. 13 and 14. In contrast to the synthetic images, where no particle images were located below the wall, mirrored images from above are present in this experiment and result in a mirrored velocity profile. However, these data points are easy to exclude from the fit, and they can even be used to determine the position of the wall reliably (Kähler et al. 2006). The resolution in the wall-normal direction is $y^{+} \approx$ 0.33 wall units and can be further increased using more images.

In order to estimate the mean flow gradient $\partial \bar{u} / \partial y$ at the wall, a linear fit-function was used, whereas at first, the wall position was determined from the symmetry plane of the near-wall profile and the mirrored profile, and secondly, the gradient was estimated using five neighboring

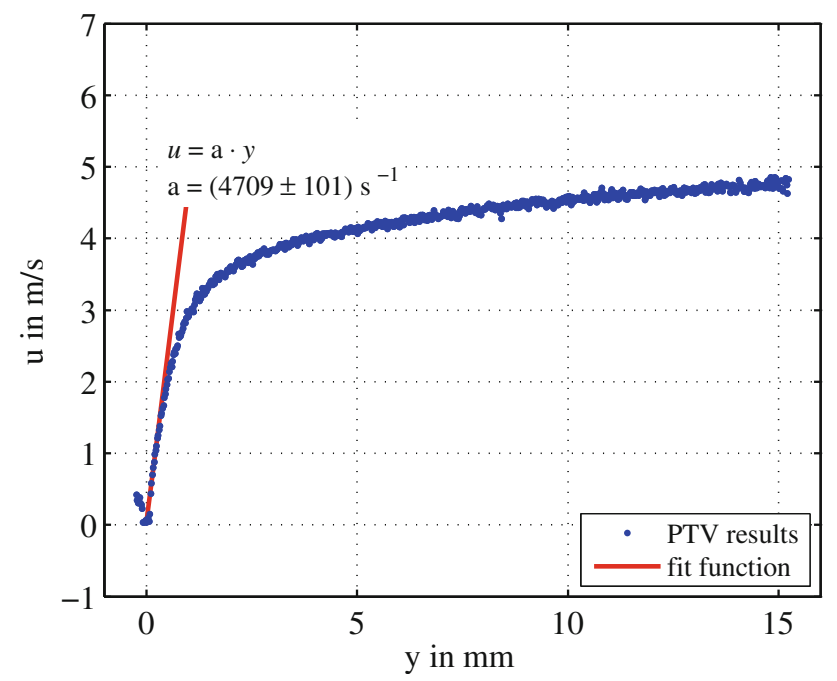

Fig. 13 Estimated velocity profile in the near-wall region of a turbulent boundary layer using long-range micro-PTV with a resolution of res $=20 \mu \mathrm{m}$ and a dynamic spatial range of $\mathrm{DSR}=750$ in the direction normal to the wall

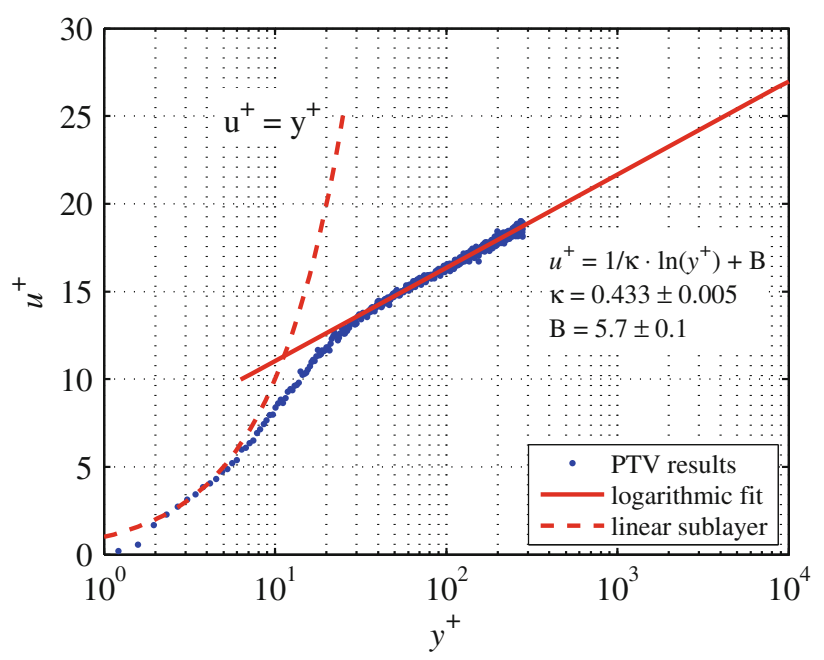

Fig. 14 Normalized velocity profile of a turbulent boundary layer from Fig. 13 normalized with $u_{\tau}=0.256 \mathrm{~m} / \mathrm{s}$. The logarithmic region was approximated by a fit-function

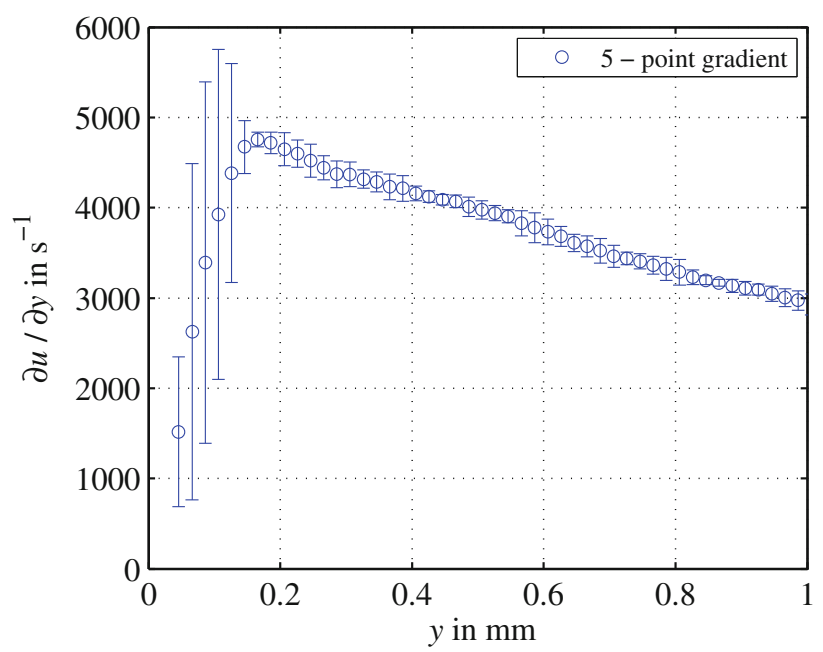

Fig. 15 Estimated velocity gradient using five points of the profile from Fig. 13 as a function of the wall distance

measurement points. Figure 15 shows the resulting gradient with respect to the wall distance of the center point (the third out of five). The error bar indicates the $95 \%$ confidence interval of the fit parameters. A maximum gradient of $\partial u / \partial y=(4,709 \pm 101) \mathrm{s}^{-1}$ was found at a wall distance of $y \approx 0.17 \mathrm{~mm}$. The profile becomes less steep further away from the wall, whereas closer to the wall, the gradient is smaller again and shows a large random error indicated by the error bar in Fig. 15. A lower limit of the friction velocity can be determined from the maximum gradient: $u_{\tau}>(0.256 \pm 0.005) \mathrm{m} / \mathrm{s}$. This agrees very well with the Clauser method, which results in the same value $u_{\tau}=0.256 \mathrm{~m} / \mathrm{s}$ for $\kappa=0.43$ and $B=5.7$ based on the logarithmic region in Fig. 14. However, the difference compared to the results obtained with the single-pixel 
method in Fig. 12 is obvious and illustrates the need to estimate $u_{\tau}$ directly with high precession nicely.

Figure 14 shows the velocity profile in normalized coordinates, where the usual normalization was used, that is $y^{+}=y \cdot u_{\tau} / v$ and $u^{+}=\bar{u} / u_{\tau}$ with the kinematic viscosity $v$. The logarithmic region, $30 \leq y^{+} \leq 200$, was approximated by an exponential equation shown in Fig. 14. The von Kármán parameters, $\kappa$ and B, served as dependent variables. The estimated values are in agreement with the range presented in the literature (Zanoun et al. 2003).

\subsection{Microscopic DPIV}

In order to validate the different evaluation methods for microscopic flow applications, an experiment in a straight micro channel was performed. The channel was made from elastomeric polydimethylsiloxane (PDMS) on a 0.6- $\mathrm{mm}$ thick glass plate with a cross-section of $514 \times 205.5 \mu \mathrm{m}^{2}$. A constant flow rate was generated by pushing distilled water through the channel using a high-precision neMESYS syringe pump (Cetoni $\mathrm{GmbH}$ ). The flow in the channel was homogeneously seeded with polystyrene latex particles with a diameter of $2 \mu \mathrm{m}$ (Microparticles $\mathrm{GmbH}$ ). The particle material was pre-mixed with a fluorescent dye and the surface was later PEG modified to make them hydrophilic. Agglomeration of particles at the channel walls can be avoided by this procedure, allowing for longduration measurements without cleaning the channels or clogging.

For the illumination, a two-cavity frequency-doubled Nd:YAG laser system was used. The laser was coupled with an inverted microscope (Zeiss Axio Observer) by an optical fiber. The image recording was performed with a 20x magnification ojective (Zeiss LD Plan-Neofluar, $\mathrm{NA}=0.4)$ using a 12-bit, 1,376 × 1,040 px, interline transfer CCD camera (PCO Sensicam QE) in doubleexposure mode. With the relay lens in front of the camera, the total magnification of the system was $M=12.6$. The time delay between the two successive frames was set to $\Delta t=100 \mu \mathrm{s}$. 8,000 image pairs were recorded at a depth of $z=93 \mu \mathrm{m}$ with an intermediate seeding concentration of $5 \times 10^{-5}$ in-focus particles per pixel, which corresponds to $0.4 \%$ of the sensor area covered by particle images or approximately 70 particles per frame. The mean in-focus digital particle image diameter was around $10 \mathrm{px}$.

Figure 16 shows the theoretical Poiseuille flow profile together with the estimated one using single-pixel ensemble-correlation as well as PTV. Since window-correlation was already proven to be erroneous due to the large averaging region, it is not considered here. To compare both methods, the PTV data were averaged using a wall-normal spatial binning size of $1 \mathrm{px}$. Using this binning size, 45

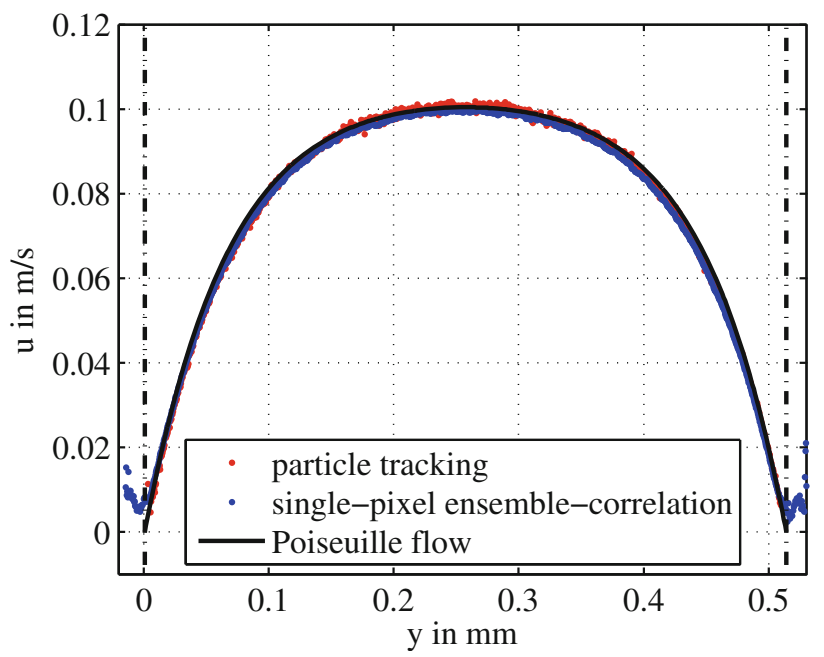

Fig. 16 Estimated velocity profiles of a laminar micro channel flow using single-pixel ensemble-correlation and PTV

particle pairs contribute on average to a single point in Fig. 16. However, close to the wall, the seeding is sparse and only several particle pairs were found. Nevertheless, the number of particle image pairs already reaches 40 at $y \approx 5 \mu \mathrm{m}$ away from the wall. Although the profile for the single-pixel ensemble-correlation is sampled on each pixel, the real spatial resolution is given by the minimum distance between independent vectors, which is $>7 \mathrm{px}$ for $D=10$ px according to Kähler et al. (2012). Thus, the spatial resolution is around $3.5 \mu \mathrm{m}$ for single-pixel ensemble-correlation and approximately $0.5 \mu \mathrm{m}$ for PTV. The corresponding values for the dynamic spatial range are $\mathrm{DSR}_{\mathrm{SP}} \approx 150$ and $\mathrm{DSR}_{\mathrm{PTV}} \approx 1,030$ independent velocity vectors in the wall-normal direction.

Due to the large difference in spatial resolution, the aforementioned bias errors for the single-pixel ensemblecorrelation can be seen on the profiles close to the wall as discussed in Sect. 3.2. As a result of the relatively large digital particle image diameter, the wall-parallel velocity at the wall is slightly overestimated as shown in the upper part of Fig. 17.

In the lower part of Fig. 17, the bias error for the wallnormal component, caused by the nonuniform seeding concentration at the wall, can be seen. An artificial velocity component toward the channel center is observed in case of single-pixel ensemble-correlation. It should be emphasized that the apparent motion of the seeding particles toward the channel's center is a pure systematic error of the evaluation approach, according to Fig. 5, and not a result of the Saffman effect (Saffmann 1965), which describes a lift force of spherical particles in a shear flow. This is evident from the PTV analysis that does not show any motion away from the wall. 

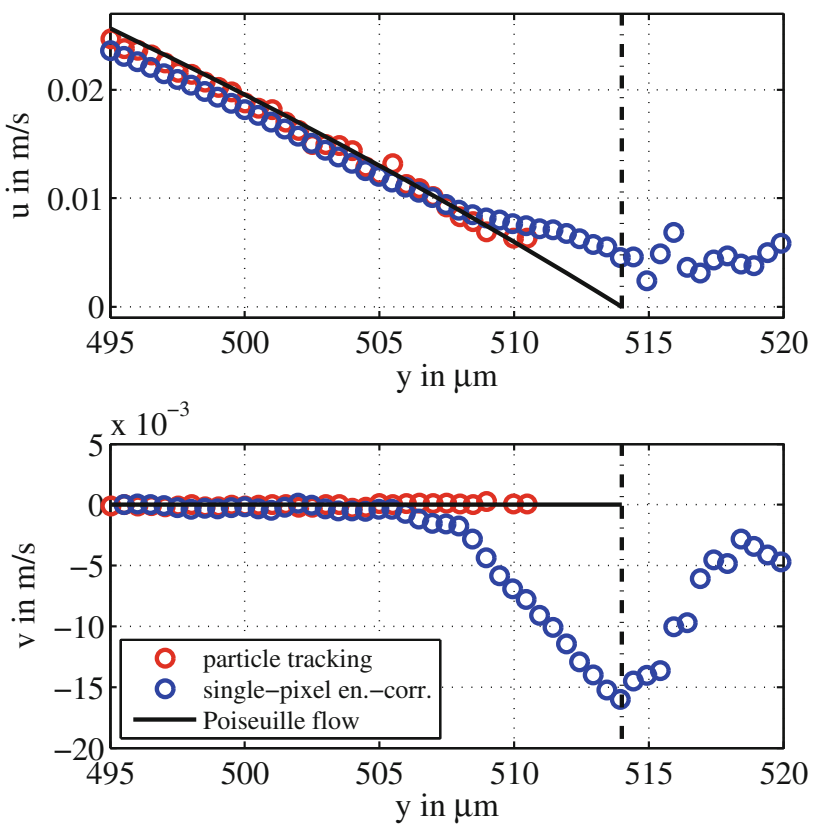

Fig. 17 Near-wall region of a laminar micro channel flow evaluated with single-pixel ensemble-correlation and PTV. Stream-wise $u$-component (top) and wall-normal $v$-component (bottom)

The theoretical flow profile in a rectangular channel is given by a series expansion of trigonometric functions (Bruus 2008). To estimate the wall gradient, the profiles were fitted by a sixth-order polynomial resulting in a wallshear stress of $\tau_{w}=0.0131 \pm 0.0002 \mathrm{~N} / \mathrm{m}^{2}$ for singlepixel ensemble-correlation and $\tau_{w}=0.0147 \pm 0.0002 \mathrm{~N} / \mathrm{m}^{2}$ for particle tracking, while the theoretical value is $\tau_{w}=0.0158 \pm 0.0001 \mathrm{~N} / \mathrm{m}^{2}$, where the uncertainty is estimated from the $95 \%$ confidence level of the fit parameters. However, the paper in hand enables to judge, to which distance the values close to the wall are biased. Using only the data points in the range of $8 \mu \mathrm{m}<y<$ $506 \mu \mathrm{m}$ results in $\tau_{w}=0.0143 \pm 0.0001 \mathrm{~N} / \mathrm{m}^{2}$ for the single-pixel ensemble-correlation. From the difference between the experimental and theoretical results, it cannot be concluded that the measurements are biased. More likely, the theoretical fitting is erroneous as the real channel geometry may differ from the assumed one. The same holds for the flow rate. For such a simple geometry, where the biased region can be properly determined, the estimation of the wall-shear stress using both methods works quite well. For more complex geometries or when a distinct wall-normal velocity is present, this is not the case.

Since the whole channel is illuminated in micro fluidics, out-of-focus particles also contribute to the correlation and bias the velocity estimate (Olsen and Adrian 2000; Rossi et al. 2011). If a normalized correlation is used, this bias is larger due to the sparse seeding, for details we refer to Cierpka and Kähler (2012). Since in-focus particles show higher intensity values, they contribute much more to the correlation peak in single-pixel evaluation and the bias due to the depth of correlation is decreased. However, the velocity is still underestimated as can be seen in Fig. 16. For the PTV evaluation, the particle image size was evaluated as well and the velocity estimation was performed later using only in-focus particles. As can be seen in Fig. 16, the velocity profile is closer to the theoretical one.

\section{Summary}

The measurement of mean quantities such as velocity profiles or Reynolds stresses is of paramount importance for the verification of theories, the validation of numerical flow simulations and the analysis of complex flows. In order to correctly measure mean quantities, the spatial resolution, dynamic spatial range and uncertainty of a measurement technique is of major relevance. In Kähler et al. (2012), it was shown that the spatial resolution of window-correlation, single-pixel ensemble-correlation and PTV approaches is limited by the following:

- interrogation window dimension $W_{Y}$ (window-correlation analysis)

- particle image diameter $D$ (single-pixel ensemblecorrelation)

- uncertainty in the estimation of the particle image position $\sigma_{\mathrm{PTV}}(\mathrm{PTV})$.

In Figs. 6 and 7 of this paper, it is shown that the measurement uncertainty of PTV can be below 0.01 pixel for low seeding densities. This is a result of the high signalto-noise ratios, which can be easily achieved experimentally, and the relative large digital particle image diameters ( $3 \mathrm{px}<D<15 \mathrm{px}$ ) that allow for a precise detection of the intensity maximum. In addition, the noise of the digital camera is uncorrelated from pixel to pixel. On the other hand, a SNR $>5$ is difficult to achieve for window-correlation analysis (if 6-8 particle images are considered for the correlation). Furthermore, the noise induced by the correlation of nonpaired particle images is correlated over a distance $\sim D$. This enhances the random errors as the interference of the signal peak with a noise peak is likely to happen.

As a consequence of these findings, the dynamic spatial range (DSR), which indicates the bandwidth or the range of scales that can be resolved on average (assuming a camera sensor with 4,000 pixel in one direction), is

- $\approx 250$ for window-correlation with $16 \times 16$ pixel interrogation windows,

- $\quad \approx 2,000$ for single-pixel ensemble-correlation with 2-3 pixel particle image diameters and 
- $\quad>25,000$ for PTV in case of low seeding densities and high signal-to-noise ratios.

To evaluate the uncertainty of mean quantities, a detailed analysis was performed. In the case of homogeneous seeding distribution and constant flow gradients, it is shown that the bias errors caused by the gradient are averaged out and window-correlation, single-pixel ensemble-correlation and PTV show identical results far away from walls (Fig. 9).

Close to walls, a bias error appears even under the same conditions (homogeneous seeding, constant flow gradient) which is given by:

- $\delta \Delta X_{\text {wall }}^{*}=W_{Y} / 4 \cdot \partial \Delta \bar{X}^{*} / \partial Y^{*}$ at the wall $\left(Y^{*}=0\right)$ and decreases to zero at $Y^{*}=W_{Y} / 2$ (window-correlation),

- $\delta \Delta X_{\text {wall }}^{*}=D /(4 \cdot \sqrt{\pi}) \cdot \partial \Delta \bar{X}^{*} / \partial Y^{*}$ at the wall $\left(Y^{*}=0\right)$ and decreases to zero at $Y^{*}=D / 2$ (single-pixel ensemble-correlation) and

- $\delta \Delta X_{\text {wall }}^{*} \approx \sigma_{\mathrm{PTV}}$ for $Y^{*}<\sigma_{\mathrm{PTV}}$ (PTV, see first point in Fig. 10).

As $\sigma_{\mathrm{PTV}} \ll D /(4 \sqrt{\pi}) \ll W_{y} / 4$, it can be concluded that the nearest unbiased measurement point for window-correlation can be increased by an order of magnitude using single-pixel ensemble-correlation, and more than an extra order of magnitude can be achieved using PTV image analysis techniques.

Furthermore, it can be concluded that PTV image analysis techniques should always be used in the case of inhomogeneous seeding and/or nonconstant flow gradients since correlation-based methods are always biased under these conditions.

This implies that the instantaneous estimation of the particle image displacement from a single image pair, calculated using window-correlation techniques, is always biased in the case of flow gradients (even for constant ones) since the particle image distribution cannot be homogeneous for a small number of randomly distributed particle images. In effect, the averaging of window-correlation results obtained from a set of image pairs leads to a larger uncertainty compared to the single-pixel ensemble-correlation analysis. This happens because the bias error due to the inhomogeneous particle image distribution appears as an increased random error on average.

For this reason, it can be concluded that the single-pixel ensemble-correlation should be used instead of windowcorrelation approaches for the estimation of averaged flow quantities at high seeding densities. However, since the particle images further away from the wall broaden the correlation peak only on the side facing away from the wall (see Fig. 3), $\Delta Y^{*}$ is also biased for $Y^{*}<D / 2$ in the case of single-pixel ensemble-correlation. Therefore, PTV should always be used near walls. This is usually possible as the particle image density decreases toward walls down to an acceptable level for an accurate PTV analysis.
Due to the high precision of the PTV evaluation technique in flows with strong gradients and inhomogeneous seeding, this approach can be even more accurate than LDV for the following reasons:

- Although the velocity estimation with LDV is usually more precise than particle imaging techniques, the localization of the measurement volume in physical space is seldom better than a fraction of a millimeter [or around $60 \mu \mathrm{m}$ for sophisticated approaches under ideal conditions (Czarske 2000)], while in PTV, it is only a few micrometers or even less for high-magnification imaging.

- In the case of flows with gradients, this position error causes significant bias errors for LDV or other measurement probes, similar to that present in the window-correlation results shown in Fig. 9.

- Uncertainties raising from mechanical translation stations or thermal elongation do not need to be considered for PTV, while they must for LDV or other probes that are traversed to measure a profile.

- Errors due to model or equipment vibrations can be completely accounted for in case of particle imaging techniques, while for LDV and other probes, this becomes difficult. In effect, the spatial resolution is further reduced using these single-point techniques.

In summary, the analysis shows that all the evaluation techniques considered in this article have their specific strengths. Therefore, a hierarchical evaluation concept that puts together the benefits of all techniques is desirable to achieve the best possible results. Unfortunately, PTV requires low particle image densities, while window-correlation and single-pixel ensemble-correlation perform best for high seeding concentrations, as this leads to small interrogation windows or moderate number of image pairs for the single-pixel ensemblecorrelation. For this reason, the development of PTV image analysis techniques with low uncertainty at high particle image densities is necessary.

Acknowledgments Financial support from German Research Foundation (DFG) in the framework of the Collaborative Research Centre-Transregio 40 and the Individual Grants Programme KA $1808 / 8$ is gratefully acknowledged by the authors. The authors also would like to thank Rodrigo Segura for technical language revisions.

Open Access This article is distributed under the terms of the Creative Commons Attribution License which permits any use, distribution, and reproduction in any medium, provided the original author(s) and the source are credited.

\section{References}

Adrian RJ (1997) Dynamic ranges of velocity and spatial resolution of particle image velocimetry. Meas Sci Tech 8:1393. doi: 10.1088/0957-0233/8/12/003 
Adrian RJ, Westerweel J (2010) Particle image velocimetry. Cambridge University Press, Cambridge

Alfredsson PH, Örlü R, Schlatter P (2011) The viscous sublayer revisited exploiting self-similarity to determine the wall position and friction velocity. Exp Fluids 51:271-280. doi:10.1007/ s00348-011-1048-8

Billy F, David L, Pineau G (2004) Single pixel resolution correlation applied to unsteady flow measurements. Meas Sci Tech 15:1039-1045

Bitter M, Scharnowski S, Hain R, Kähler CJ (2011) High-repetitionrate PIV investigations on a generic rocket model in sub- and supersonic flows. Exp Fluids 50:1019-1030. doi:10.1007/ s00348-010-0988-8

Bruus H (2008) Theoretical microfluidics. Oxford master series in condensed matter physics. Oxford University Press, Oxford

Buchmann NA, Atkinson C, Jeremy M, Soria J (2011) Tomographic particle image velocimetry investigation of the flow in a modeled human carotid artery bifurcation. Exp Fluids 50:1131-1151. doi: 10.1007/s00348-011-1042-1

Burdick G, Berman N, Beaudoin S (2001) Describing hydrodynamic particle removal from surfaces using the particle Reynolds Number. J Nanoparticle Res 3:453-465. doi:10.1023/A:1012593 318108

Cardwell ND, Vlachos PP, Thole KA (2011) A multi-parametric particle-pairing algorithm for particle tracking in single and multiphase flows. Meas Sci Tech 22:105406. doi:10.1088/ 0957-0233/22/10/105406

Champagne N, Vasseur R, Montourcy A, Bartolo D (2010) Traffic jams and intermittent flows in microfluidic networks. Phys Rev Lett 105:044502. doi:10.1103/PhysRevLett.105.044502

Christensen K (2004) The influence of peak-locking errors on turbulence statistics computed from PIV ensembles. Exp Fluids 36:484-497. doi:10.1007/s00348-003-0754-2

Cierpka C, Kähler CJ (2012) Particle imaging techniques for volumetric three-component (3D3C) velocity measurements in microfluidics. J Vis 15:1-31. doi:10.1007/s12650-011-0107-9

Cierpka C, Segura R, Hain R, Kähler CJ (2010) A simple single camera $3 \mathrm{C} 3 \mathrm{D}$ velocity measurement technique without errors due to depth of correlation and spatial averaging for microfluidics. Meas Sci Tech 21:045401. doi:10.1088/0957-0233/21/4/ 045401

Clauser FH (1956) The turbulent boundary layer. Adv Appl Mech $4: 1-51$

Czarske J (2000) Laser doppler velocity profile sensor using a chromatic coding. Meas Sci Tech 12:52-57. doi:10.1088/09570233/12/1/306

Dumitra M, Schanz D, Schröder A, Kähler CJ (2011) Large-scale turbulent boundary layer investigation with multiple camera PIV and hybrid evaluation up to single pixel resolution. In: 9th International symposium on particle image velocimetry, Kobe, Japan, July 21-23

Fernholz HH, Finley PJ (1996) The incompressible zero-pressuregradient turbulent boundary layer: an assessment of the data. Prog Aerospace Sci 32:245-311. doi:10.1016/0376-0421(95) 00007-0

Fincham AM, Spedding GR (1997) Low cost, high resolution DPIV for measurements of turbulent flow. Exp Fluids 23:449-462. doi: $10.1007 / \mathrm{s} 003480050135$

Hain R, Kähler CJ, Tropea C (2007) Comparison of CCD, CMOS and intensified cameras. Exp Fluids 42:403-411. doi:10.1007/ s00348-006-0247-1

Kähler CJ (1997) Ortsaufgelöste Geschwindigkeitsmessungen in einer turbulenten Grenzschicht. DLR-FB:97-32

Kähler CJ, Scholz U (2006) Transonic jet analysis using long-distance micro PIV. In: 12th International symposium on flow visualization, Göttingen, Germany
Kähler CJ, Sammler B, Kompenhans J (2002) Generation and control of particle size distributions for optical velocity measurement techniques in fluid mechanics. Exp Fluids 33:736-742. doi: 10.1007/s00348-002-0492-x

Kähler CJ, Scholz U, Ortmanns J (2006) Wall-shear-stress and nearwall turbulence measurements up to single pixel resolution by means of long-distance micro-PIV. Exp Fluids 41:327-341. doi: 10.1007/s00348-006-0167-0

Kähler CJ, Scharnowski S, Cierpka C (2012) On the resolution limit of digital particle image velocimetry. Exp Fluids doi:10.1007/ s00348-012-1280-x

Keane RD, Adrian RJ (1992) Theory of cross-correlation analysis of PIV images. Appl Sci Res 49(3):191-215. doi:10.1007/BF00384623

Keane RD, Adrian RJ, Zhang Y (1995) Super-resolution particle imaging velocimetry. Meas Sci Tech 6:754-768. doi:10.1088/ 0957-0233/6/6/013

Marusic I, McKeon BJ, Monkewitz PA, Nagib HM, Smits AJ, Sreenivasan KR (2010) Wall-bounded turbulent flows at high Reynolds Numbers: recent advances and key issues. Phys Fluids 22:065103. doi:10.1063/1.3453711

Meinhart CD, Wereley ST, Santiago JG (2000) A PIV algorithm for estimating time-averaged velocity fields. J Fluids Eng 122:285289. doi: $10.1115 / 1.483256$

Melling A (1997) Tracer particles and seeding for particle image velocimetry. Meas Sci Tech 8:1406-1416. doi:10.1088/09570233/8/12/005

Nagib HM, Chauhan KA, Monkewitz PA (2007) Approach to an asymptotic state for zero pressure gradient turbulent boundary layers. Phil Trans R Soc A 365:755-770. doi:10.1098/rsta. 2006.1948

Nguyen CV, Nguyen TD, Wells JC, Nakayama A (2010) Interfacial PIV to resolve flows in the vicinity of curved surfaces. Exp Fluids 48:577-587. doi:10.1007/s00348-010-0824-1

Nickels TB (2004) Inner scaling for wall-bounded flows subject to large pressure gradients. J Fluid Mech 521:217-239. doi:10.1017/ S0022112004001788

Ohmi K, Li HY (2000) Particle tracking velocimetry with new algorithms. Meas Sci Tech 11:603-616. doi:10.1088/0957-0233/ $11 / 6 / 303$

Olsen MG, Adrian RJ (2000) Out-of-focus effects on particle image visibility and correlation in microscopic particle image velocimetry. Exp Fluids 29:S166-S174. doi:10.1007/s003480070018

Raffel M, Willert CE, Wereley ST, Kompenhans J (2007) Particle image velocimetry: a practical guide. Springer, New York

Rossi M, Lindken R, Hierck BP, Westerweel J (2009) Tapered microfluidic chip for the study of biochemical and mechanical response of endothelial cells to shear flow at subcellular level. Lab Chip 9:1403-1411. doi:10.1039/B822270N

Rossi M, Segura R, Cierpka C, Kähler CJ (2011) On the effect of particle image intensity and image preprocessing on depth of correlation in micro-PIV. Exp Fluids 52:1063-1075. doi:10. 1007/s00348-011-1194-z

Saffmann PG (1965) The lift on a small sphere in a slow shear flow. J Fluid Mech 22:385-400. doi:10.1017/S0022112065000824

Santiago JG, Wereley ST, Meinhart CD, Beebe DJ, Adrian RJ (1998) A particle image velocimetry system for microfluidics. Exp Fluids 25:316-319. doi:10.1007/s003480050235

Scarano F (2001) Iterative image deformation methods in PIV. Meas Sci Tech 13:R1-R19. doi:10.1088/0957-0233/13/1/201

Scharnowski S, Hain R, Kähler CJ (2011) Reynolds stress estimation up to single-pixel resolution using PIV-measurements. Exp Fluids 52:985-1002. doi:10.1007/s00348-011-1184-1

Scholz U, Kähler CJ (2006) Dynamics of flow structures on heaving and pitching airfoils. In: 13th International symposium on applications of laser techniques to fluid mechanics, Lisbon, Portugal 
Stanislas M, Okamoto K, Kähler CJ (2003) Main results of the first international PIV challenge. Meas Sci Tech 14:R63-R89. doi: 10.1088/0957-0233/14/10/201

Stanislas M, Okamoto K, Kähler CJ, Westerweel J (2005) Main results of the second international PIV challenge. Exp Fluids 39:170-191. doi:10.1007/s00348-005-0951-2

Stanislas M, Okamoto K, Kähler CJ, Westerweel J, Scarano F (2008) Main results of the third international PIV challenge. Exp Fluids 45:27-71. doi:10.1007/s00348-008-0462-z

Stitou A, Riethmuller ML (2001) Extension of PIV to super resolution using PTV. Meas Sci Tech 12:1398-1403. doi:10.1088/09570233/21/4/045401

Theunissen R, Buchmann NA, Corieri P, Riethmuller ML, Darquenne C (2006) Experimental investigation of aerosol deposition in alveolar lung airways. In: 13th International symposium on applications of laser techniques to fluid mechanics, Lisbon, Portugal, June 26-29

Theunissen R, Scarano F, Riethmuller ML (2008) On improvement of PIV image interrogation near stationary interfaces. Exp Fluids 45:557-572. doi:10.1007/s00348-008-0481-9
Wereley S, Whitacre I (2007) Particle dynamics in a dielectrophoretic microdevice. BioMEMS Biomed Nanotechnol 3:259-276. doi: 10.1007/978-0-387-25845-4-13

Wernet JH, Wernet MP (1994) Stabilized alumina/ethanol colloidal dispersion for seeding high temperature air flows. In: Proceedings of the ASME symposium on laser anemometry: advances and applications, Lake Tahoe, NV, June 19-23

Westerweel J, Geelhoed PF, Lindken R (2004) Single-pixel resolution ensemble correlation for micro-PIV applications. Exp Fluids 37:375-384. doi:10.1007/s00348-004-0826-y

Willert C (1996) The fully digital evaluation of photographic piv recordings. Appl Sci Res 56:79-102. http://dx.doi.org/10.1007/ BF02249375, 10.1007/BF02249375

Zanoun ES, Durst F, Nagib H (2003) Evaluating the law of the wall in two-dimensional fully developed turbulent channel flows. Phys Fluids 15:3079-3089. doi:10.1063/1.1608010 